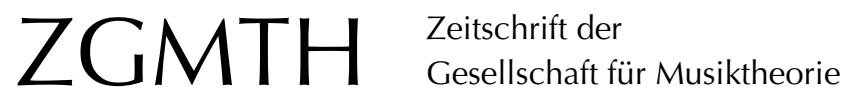

Kunkel, Liselotte (2010): Harmonisches Hören im Stilbereich Impressionismus. ZGMTH 7/2, 179-201. https://doi.org/10.31751/510

C 2010 Liselotte Kunkel

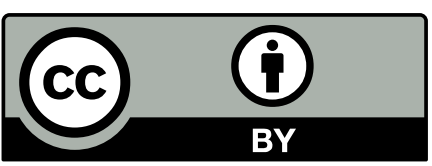

Dieser Text erscheint im Open Access und ist lizenziert unter einer Creative Commons Namensnennung 4.0 International Lizenz.

This is an open access article licensed under a

Creative Commons Attribution 4.0 International License.

veröffentlicht / first published: 05/07/2010

zuletzt geändert / last updated: 01/09/2010 


\title{
Harmonisches Hören im Stilbereich Impressionismus
}

\author{
Lilo Kunkel
}

Der vorliegende Text zeigt Möglichkeiten zur Erarbeitung des Stilbereichs ^Impressionismus` im Gehörbildungsunterricht. Dabei werden anhand zahlreicher Literaturbeispiele, die jeweils eine systematische Analyse erfahren, zentrale Kompositionstechniken erläutert.

\section{EINLEITUNG}

Musiktheorie und musiktheoretischer Unterricht haben den musikalische Impressionismus noch nicht angemessen erschlossen. Begrifflichkeiten wie "Ganztondiatonik« oder die Behauptung, dass »Quartenklänge anstelle von Terzschichtungen « aufträten, spiegeln die Unklarheiten, die nach wie vor bestehen. Erst recht wird im Gehörbildungsunterricht selten auf typisch impressionistische Strukturen eingegangen. Dabei wäre hier wie auch in anderen Stilbereichen eine gezielte Hörschulung von großer Bedeutung:

- Strukturelles Hören und das Unterscheiden verschiedener tonsetzerischer Fakturen geben Aufschluss über die Großform und übergeordnete Gestaltungsprinzipien eines Stückes und gegebenenfalls über programmatische Zusammenhänge.

- Je genauer eine Struktur (beispielsweise in der Literatur für den instrumentalen Hauptfachunterricht) bereits hörend erschlossen wird, desto leichter kann sie gezielt geübt werden. Das Auswendiglernen und Auffinden von Fehlern wird erleichtert.

- Schließlich ist das genaue hörende (und analytische) Erfassen Voraussetzung, um bestimmte Klanglichkeiten improvisatorisch nachzuvollziehen zu können.

Viele impressionistische Strukturen sind sehr komplex. Gerade im üppig instrumentierten, vielschichtigen orchestralen Satz und bei schnellen Tempi ist das hörende Erfassen eine anspruchsvolle Aufgabe. Andere, häufig vorkommende Fakturen und Tonalitäten wiederum eignen sich sehr gut für Gehörbildungsaufgaben. Dabei verbindet sich das Erfassen des impressionistischen Stilbereichs mit dem Hören elementarer Klanglichkeiten (Pentatoniken, Vierklänge im Bereich der sogenannten Mixturen etc.) und der Wiederholung von Klängen, die bereits aus der sromantischen (Musik bekannt sind bzw. sein sollten (z. B. Mehrklänge mit typischen Hinzufügungen). Anhand der Préludes von Debussy

1 Bieri 1935, 183. 
werden im Folgenden einige mögliche Aufgabenstellungen beschrieben. Die römische Ziffer bezeichnet den Band (I oder II) der Préludes, die arabische Ziffer die Nummer des Stückes im Zyklus (jeweils 1-12); danach folgt die Angabe der jeweils relevanten Takte (manche der zitierten Notenbeispiele geben darüber hinaus einen größeren Zusammenhang wieder).

Die analytischen Begrifflichkeiten orientieren sich sich an der Harmonik von Gárdonyi/Nordhoff, die an die ungarische Musiktheorie-Tradition (etwa die Bartók-Forschung von Ernö Lendvai) anknüpft und wertvolle Kategorien insbesondere für die Analyse der Klangsprache um 1900 zur Verfügung stellt.

\section{TONVORRÄTE}

Im Impressionismus etablieren sich Tonvorräte, die teilweise schon in der Romantik auftreten, als selbstverständliches Material. So kann man etwa aus der figurativen Akkorderweiterung ${ }^{2}$ die Entstehung der sogenannten akustischen Tonalität ableiten. Diese und andere auch verselbständigt auftretende Tonsysteme sollen im Folgenden besprochen werden.

\subsection{Pentatonik}

\section{Analytische Vorbemerkung}

Pentatonische Felder treten in der impressionistischen Musik in verschiedenen satztechnischen Erscheinungsformen auf, beispielsweise als Melodie, als arpeggierter $`$ Klangteppich oder in Form von akkordischen `Auftragungen . Diether de la Motte erklärt ihr Vorkommen als eine der im zwölf- und gleichstufig temperierten chromatischen Tonsystem möglichen Annäherungen an die indonesische Materialtonleiter Slendro, die »die Oktave in fünf nahezu aber nicht exakt gleiche Teile« teilt, "wobei zwei Distanzen etwas größer sind als die übrigen drei . $^{3}$ Auch werden Pentatoniken, vor allem pentatonische Melodien, oft in sarchaisierendem Zusammenhang eingesetzt.

Die hörende Erschließung von pentatonischen Feldern gibt nicht nur Aufschluss über impressionistische Kompositionstechniken, sondern bildet auch eine gute Grundlage für elementare Hör- und Singübungen. Erzsébet Hegyi begründet dies in den methodischen Handreichungen zur Arbeit mit der Kodály-Chorschule folgendermaßen:

[...] the melodic patterns of this pentatonic system are extremely clear, even their great variety does not preclude simplicity, for their intervals do not include any diminished or augmented interval or the upward or downward minor second. For precisely these reasons varied vocal practice in pentatony can lay the foundations of secure intonation. ${ }^{4}$

2 Gárdonyi/Nordhoff 1990, $116 \mathrm{ff}$. und $135 \mathrm{ff}$.

3 La Motte 1976, 249. Die Stimmung von Gamelan-Instrumenten unterscheidet sich allerdings grundlegend von der gleichstufigen Temperatur. Vgl. hierzu Schumacher u. a. 1986, 782, insbes. Sp. 1 und 2.

4 Hegyi 1975, 18. 
Neben Singeübungen im pentatonischen Raum, zu denen die Kodály-Schule zahlreiche Anregungen bietet, sind solche Übungen wichtig, bei denen sich das Erfassen pentatonischer Strukturen mit der analytischen Erkenntnis über Grund- bzw. Bezugstöne und dem Gebrauch der entsprechenden Nomenklatur verbindet. Benennt man den Ton einer pentatonischen Skala, der dem Grundton der entsprechenden Durskala entspricht, mit der Solmisationssilbe >Do‘, ergeben sich für die anderen Töne der Pentatonik die Namen >Res, >Mis, >Sor und ‘Las.

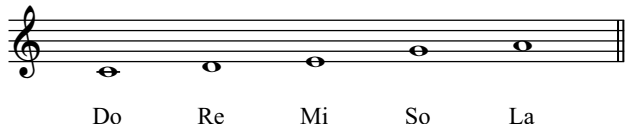

Beispiel 1: C-Do-Pentatonik nach Gárdonyi/ Nordhoff 1990, 224

Do $\quad \operatorname{Re} \quad \mathrm{Mi} \quad$ So $\quad \mathrm{La}$

Neben der elementaren Skala c- $d$-e-g-a (C-Do-Pentatonik) können auch pentatonische Skalen oder Felder mit anderem Grundton solmisatorisch präzisiert werden. (Als Grundton kann man dabei beispielsweise den tiefsten Ton eines pentatonischen Akkords oder die Finalis einer pentatonischen Melodie auffassen.)

a) C-So-Pentatonik

b) C-Mi-Pentatonik

c) C-Re-Pentatonik

d) C-La-Pentatonik

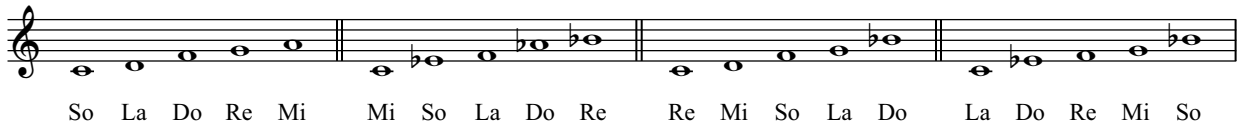

Beispiel 2: Verschiedene Pentatoniken über c nach Gárdonyi/Nordhoff 1990, $224 \mathrm{f}$.

Zwei Übungen lassen sich daraus ableiten, die gleichzeitig den Überblick über verschiedene Vorzeichenbereiche schulen:

1. Von einem gewählten Grundton aus (vgl. Beispiel 3: Bezugston es) wird die zugehörige Do-Pentatonik ermittelt (Vorzeichenbereich 3b), dann (eventuell unter Nachkontrolle durch das Klavier) sowohl mit absoluten Tonnamen als auch relativen Solmisationssilben gesungen. Dazu erzeugt man die Parallelskalen (also Skalen mit gleichem Tonvorrat, aber anderem Grundton, z.B. F-Re-, G-Mi-Pentatonik etc.) in gleicher Weise. Alle so entstehenden Skalen lassen sich als der ^3-b-Pentatonikı zugehörig bezeichnen.

2. Von einem gleichbleibenden Grundton aus werden alle möglichen Pentatoniken gesungen (Beispiel 4: F-Do $=1 \mathrm{~b}, \mathrm{~F}-\mathrm{Re}=3 \mathrm{~b}, \mathrm{~F}-\mathrm{Mi}=5 \mathrm{~b}$ etc.).

a) Es-Do-Pentatonik

b) F-Re-Pentatonik

c) G-Mi-Pentatonik

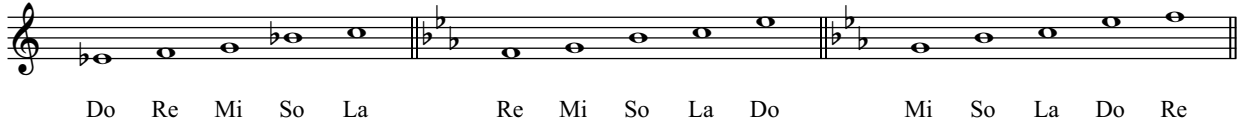

Beispiel 3: Es-Do-Pentatonik und Parallelskalen 
a) F-Do-Pentatonik

b) F-Re-Pentatonik

C) F-Mi-Pentatonik

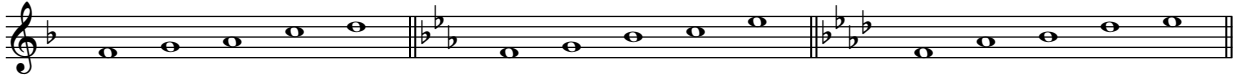

d) F-So-Pentatonik

e) F-La-Pentatonik

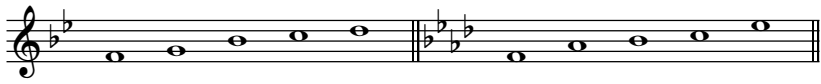

Beispiel 4: Pentatoniken über einem gleichbleibenden Grundton

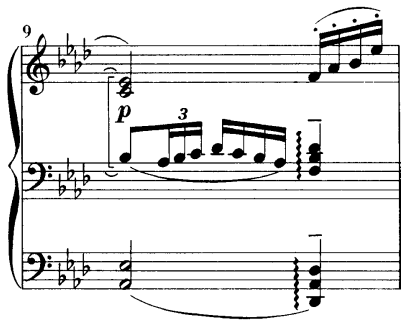

Beispiel 5: Claude Debussy, Préludes II, Nr. 5, T. 9
Solche Übungen können dazu beitragen, dass Pentatoniken mit der Zeit hörend sabsolut erkannt werden. Die Identifikation eines Partiturausschnitts als pentatonisch wird oft dadurch erleichtert, dass das Notenbild in vielen Fällen sverräterisch lückenhaft, nämlich squartigs oder sterzigı, erscheint, so bei der Des-Do-Pentatonik in Beispiel 5 (II, 5, T. 9, 3. Viertel). Der smilde` oder dissonanzarme Klangeindruck pentatonischer Felder ist ein weiteres Erkennungsmerkmal.

\section{Pentatonische Melodien}

Beispiel 6 (II, 9, T. 44 ff.) eignet sich gut als Einstiegsübung zur Erschließung pentatonischer Melodien, da hier die (kurzzeitige) Etablierung des neuen Tonvorrats mit einem sscharfkantigen` Fakturwechsel im Klaviersatz zusammenfällt und daher leicht wahrnehmbar ist.

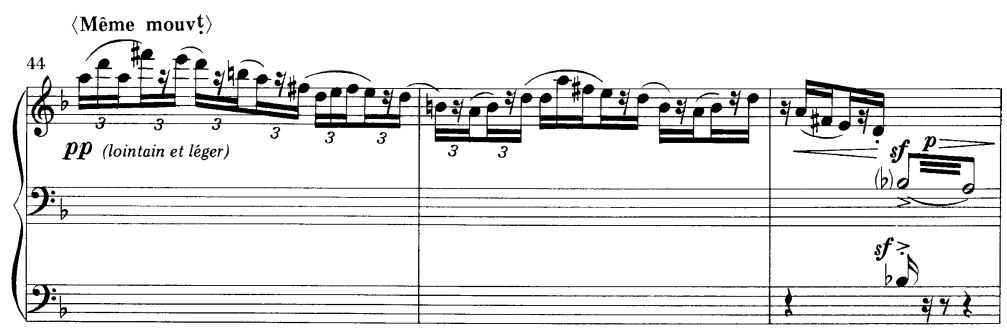

Beispiel 6: Claude Debussy, Préludes II, Nr. 9, T. 44-46

Isoliert man die pentatonischen Takte aus dem Zusammenhang, kann man daran im langsamen Tempo verschiedene Übungen vornehmen:

Das >Do zu finden, wird beim langsamen Spielen bzw. Singen keine besondere Mühe bereiten, da durch die großenteils skalare Struktur der Melodie ein deutlicher Grundtonbezug vorliegt. Die wenigen Stellen, an denen die Skala durchbrochen ist, er- 
fordern besonderes Augenmerk beim solmisierten Singen. Indem man die Studierenden von verschiedenen Stellen des Ausschnittes an singen lässt (z. B. ab T. 45), kann die Sicherheit im Auffinden der richtigen Silben überprüft werden.

Interessant ist der unterschiedliche ssinnliche` Gehalt, den Pentatoniken je nach Konstellation annehmen können. Für den weniger geübten Hörer ist es wichtig, nicht nur »durale $«^{5}$ Ausschnitte wie das obige Notenbeispiel, sondern auch smollare Melodien wie Beispiel 7 (II, 10, T. 5 f., Viertelnoten) als pentatonisch identifizieren zu können.

Zur analytischen Erschließung sollen zunächst wieder das `Do $<$ und davon ausgehend die anderen Solmisationssilben aufgefunden und singend geübt werden. Dazu kann man am Klavier ein pentatonisches Total anschlagen und die Töne gezielt hineinsingen bzw. heraushören. Das Ohr wird beim Hören des Ausschnittes entweder einer D-Re-Pentatonik (wegen des vorherigen tonikalen d-Moll-Dreiklangs) oder einer A-La-Pentatonik (wegen der Vierklangsbrechung a-c-e-g, die etwa halbtaktig zu hören ist) zuneigen; in beiden Fällen ergibt sich ein mollarer Klangeindruck.

Den sschwebenden ‘ Charakter der So-Pentatonik kann man an Beispiel 8 (I, 5, T. 1-2) wahrnehmen, das ebenfalls durch solmisiertes Singen erschlossen werden soll. Beide vorausgegangenen Ausschnitte bieten sich für die weiterführende Hörübung an, Pentatoniken auch innerhalb der typisch impressionistischen Schichtstrukturen (z. B. Melodieschicht plus akkordische Begleitschicht plus Orgelpunktschicht im Instrumentalsatz) aufzufinden.

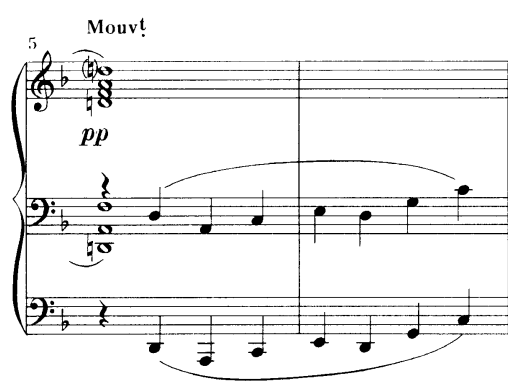

Beispiel 7: Claude Debussy, Préludes II, Nr. 10, T. 5-6

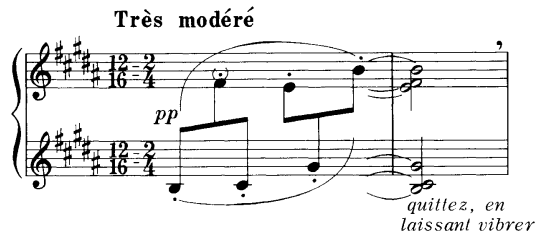

Beispiel 8: Claude Debussy, Préludes I, Nr. 5, T. 1-2

\section{Pentatonische Akkorde}

Innerhalb von Mixturen, aber auch in anderen Zusammenhängen ergeben sich oft Klänge, die sich in ihrer weiträumigen Auftragung (`weite Lage ) eignen, um vertikal erschlossen zu werden. Beispiel 9 (I, 8, T. 24-26) lässt sich als pentatonische Mixtur in der oben beschriebenen Weise singen.

5 Die Assoziation der Do-Pentatonik mit Dur ergibt sich im ersten Takt dieses Beispiels wohl aus der besonderen (metrischen) Betontheit der Töne Do-Mi-So (Dur-Dreiklang). 


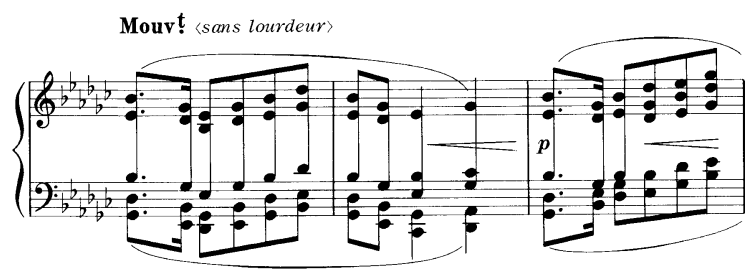

Beispiel 9: Claude Debussy, Préludes I, Nr. 8, T. 24-26

Hier bietet sich außerdem an, jeden Klang einzeln von unten nach oben solmisiert zu singen. Dass dabei der Klang oktavreduziert gesungen und die jeweils treffsicherste und bequemste Stimmlage gewählt wird, versteht sich hier wie bei vergleichbaren Singübungen von selbst. Das Hören des jeweiligen Grundtonbezuges und der Klangcharakteristik etwa einer kurzzeitigen Mi- oder La-Pentatonik (auch im nur viertönigen Akkord) hilft dabei, solche Klänge auch in anderem Zusammenhang zu erkennen.

Vollständige pentatonische Klänge ergeben sich außerhalb von Mixturen in den Beispielen 10 (II, 4, T. 24, 2. und 3. Achtel) und 11 (II, 9, T. 49). Beim erstgenannten Beispiel kann man darauf hinweisen, dass die Re-Pentatonik schon in früh- und hochromantischen Kompositionen auftritt, dort meistens als dominantischer Klang. Die üppige weite Lage der F-Do-Pentatonik in Beispiel 11 bietet Hörhilfe und Improvisations- bzw. Instrumentationsanregung etwa für den Jazz-Klaviersatz (typisches F6/9-Voicing).

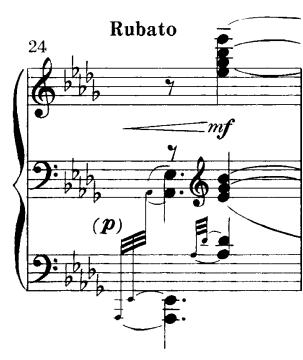

Beispiel 10: Claude Debussy, Préludes II, Nr. 4, T. 24

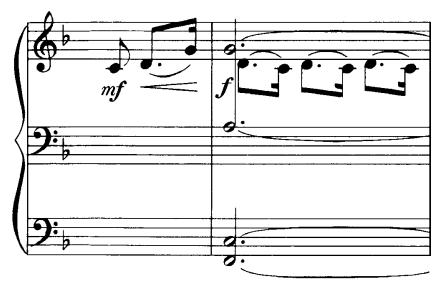

Beispiel 11: Claude Debussy, Préludes II, Nr. 9, T. 49

\section{Pentatonische Felder}

Neben pentatonischen Einzelakkorden finden sich in impressionistischen Kompositionen oft größere Abschnitte die auf pentatonischen Tonvorräten beruhen. Eine weitere Hörfähigkeit, die trainiert werden kann, besteht also darin, auch im raschen Tempo zu erfassen, dass ein pentatonisches Feld erklingt, und dieses zu bestimmen. Skalar arpeggierte Felder sind dabei am leichtesten zu erschließen. Beispiel 12 (I, 11, T. 73 und 75, jeweils 2. Viertel) kann zuerst mehrmals im Originaltempo angehört werden. Durch langsames Durchspielen und Durchsingen bestimmt man es analytisch als So-Pentatonik. Bei erneutem Hören des Originals wird diese Erkenntnis gefestigt. 


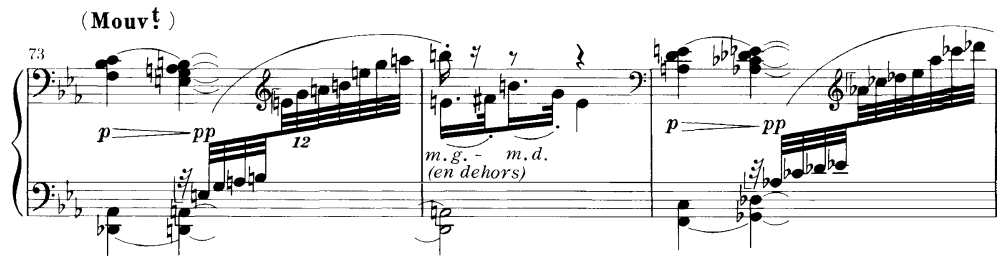

Beispiel 12: Claude Debussy, Préludes I, Nr. 11, T. 73-75

\section{Unterscheiden pentatonischer Felder von anderen Tonvorräten}

Mit der vorherigen Aufgabenstellung verwandt ist die Übung, hörend zwischen Pentatoniken und anderen Klanglichkeiten zu unterscheiden. Mit Beispiel 13 (I, 2, T. 38-48) kann die Fähigkeit geübt werden, den sinnlichen Unterschied zwischen der scharfen, ganztönigen Tonalität und der milden Pentatonik wahrzunehmen und den genauen Zeitpunkt des U Umschlagens` zu bestimmen. Der Fakturwechsel im Klaviersatz erleichtert dabei das differenzierende Hören.
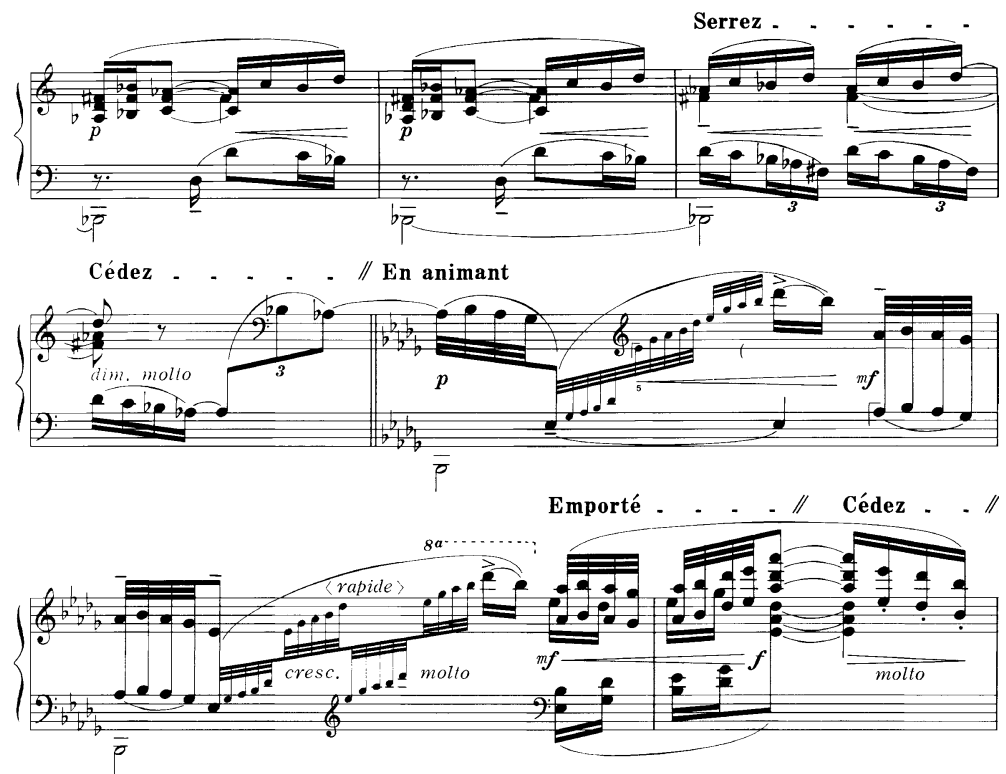

Très retenu

au Mouvt

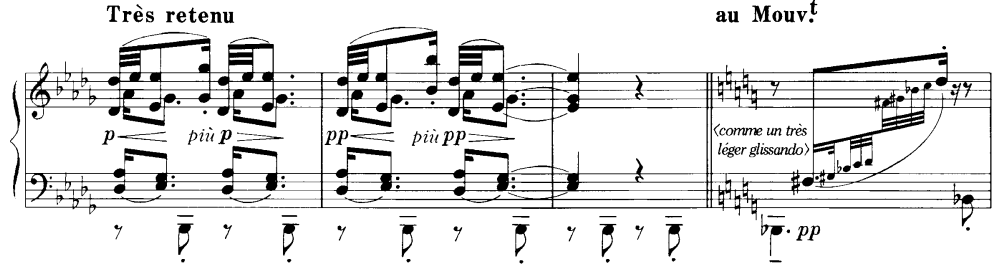

Beispiel 13: Claude Debussy, Préludes I, Nr. 2, T. 38-48 


\subsection{Akustische Tonalität}

\section{Begriffsklärung}

Das Material der akustischen Tonalität lässt sich mit einer Durskala vergleichen, in der die Töne `Fa rührt daher, dass die vorkommenden Töne die (an die chromatisch gleichstufige Temperatur angeglichenen) Partialtöne 8 bis 14 darstellen. ${ }^{6}$

a) D-Dur

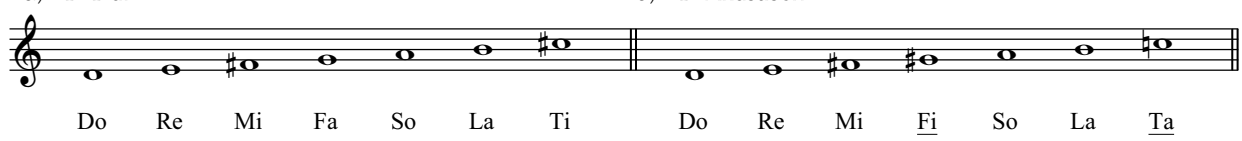

Beispiel 14: Durskala und akustische Skala

Hinsichtlich der akustischen Tonalität kann sich der noch ungeübte Hörer an dem Sachverhalt orientieren, dass die Skala jeweils sechs Töne gemeinsam mit Lydisch (außer `Ta`) und Mixolydisch (außer $>\mathrm{Fi}<$ ) hat. Auch kann die Aufteilung in eine untere ganztönige (aufwärts Ta bis Fi) und eine obere alternierend halb- und ganztönige Hälfte (aufwärts Mi bis Do) ein Anhaltspunkt beim Hören sein.

\section{Skalare Auftragung}

Als Einstiegsübung in das Hören von akustischen Feldern bietet sich das Hören und Nachsingen (solmisiert und absolut) von Skalenausschnitten an. In Beispiel 15 (II, 10, T. 13) können bei gehaltenem Akkord $\left(\mathrm{Es}^{7}\right) \mathrm{zu}-$ nächst der Skalenausschnitt abzüglich des leiterfremden Tones e gesungen, dann die fehlenden Töne der Skala ergänzt werden.

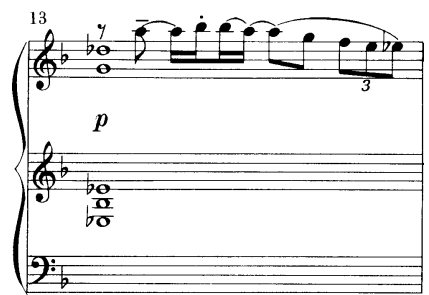

Beispiel 15: Claude Debussy, Préludes II, Nr. 10, T. 13

\section{Akkordische Auftragung}

Als Übung zum Durchhören und Erkennen akustischer Felder, die komprimierter aufgetragen sind, eignen sich die Beispiele 16 und 17. Beispiel 16 (II, 4, T. 94-100) soll zunächst als akustisch erkannt werden. Dann kann der Studierende den zugrundeliegenden Tredezimakkord arpeggiert spielen und seine Bestandteile von unten nach oben sowie in der Reihenfolge Terz, Septime, Tredezime nachsingen. Die durch den Triller hinzutretenden Akkordtöne sollen als None und Terz ebenfalls analytisch wahrgenommen und singend nachvollzogen werden. Als letzter Schritt kann man die Mittelstimme solmisiert und absolut gesungen ergänzen. 

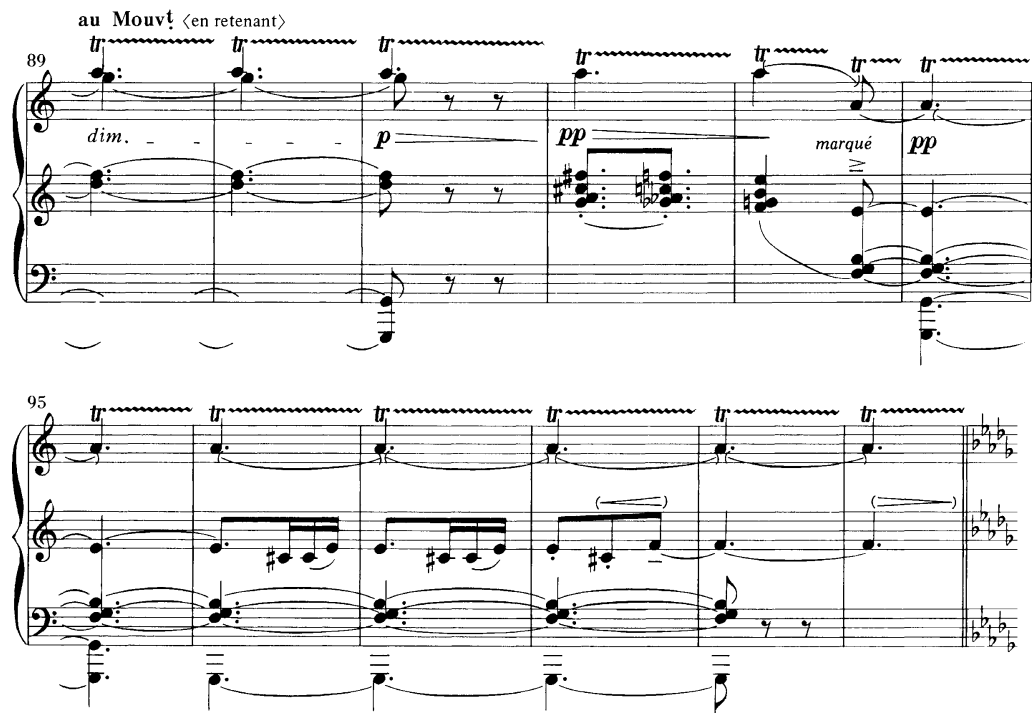

Beispiel 16: Claude Debussy, Préludes II, Nr. 4, T. 89-100
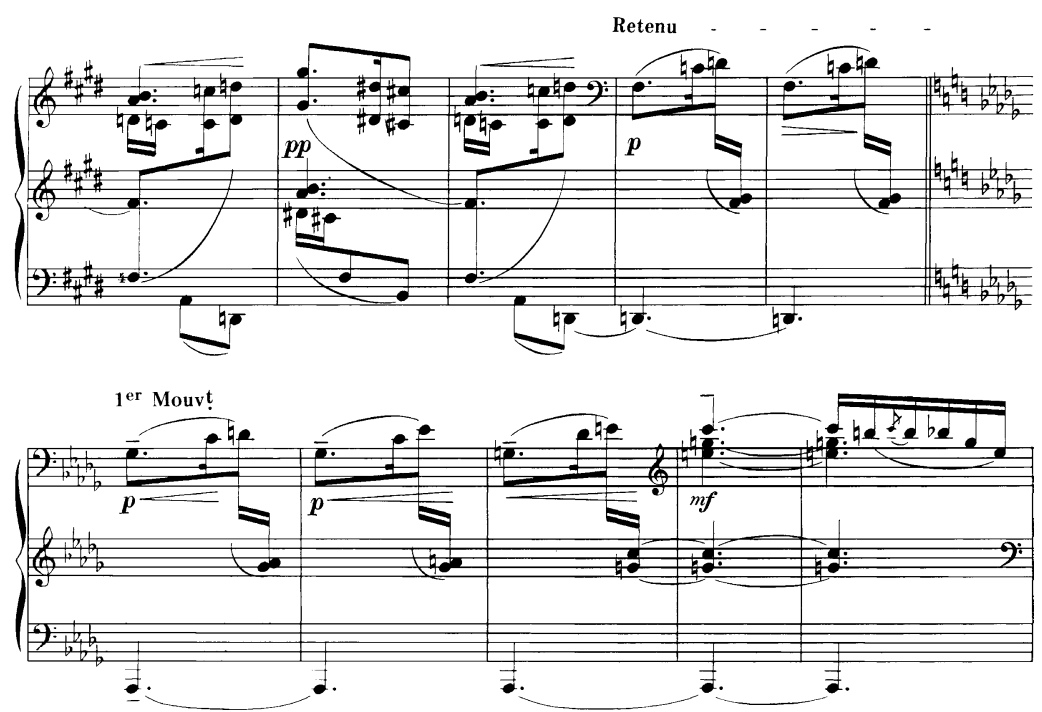

Beispiel 17: Claude Debussy, Préludes II, Nr. 4, T. 37-46

Der Undezimakkord aus dem Tonvorrat D-Akustisch bzw. As-Akustisch in Beispiel 17 (II, 4, T. 40-43) kann absolut gesungen in beiden enharmonischen Varianten, solmisiert gesungen mit as oder $d$ als Do dargestellt werden. 


\section{Unterscheiden akustischer Felder von anderen Tonvorräten}

Die Beispiele 18 und 19 bieten sich an, um die Unterscheidung zwischen der akustischen Skala und den ihr ähnelnden kirchentonalen diatonischen Skalen zu üben. Beispiel 18 (II, 8, T. 8-9) stellt Teile einer Skala dar, die je nach Ergänzung als akustisch oder mixolydisch gedeutet werden kann. Durch abwechselndes Hineinsingen des Tones e für Akustisch und es für Mixolydisch soll die genaue hörende Unterscheidung dieser Modi geübt werden. Ebenso lässt sich der indifferenteく Tonvorrat des Beispiels 19 (II, 8, T. 14) durch Ergänzung des Tones $c$ in Akustisch oder durch Ergänzung des Tones cis in Lydisch verwandeln.

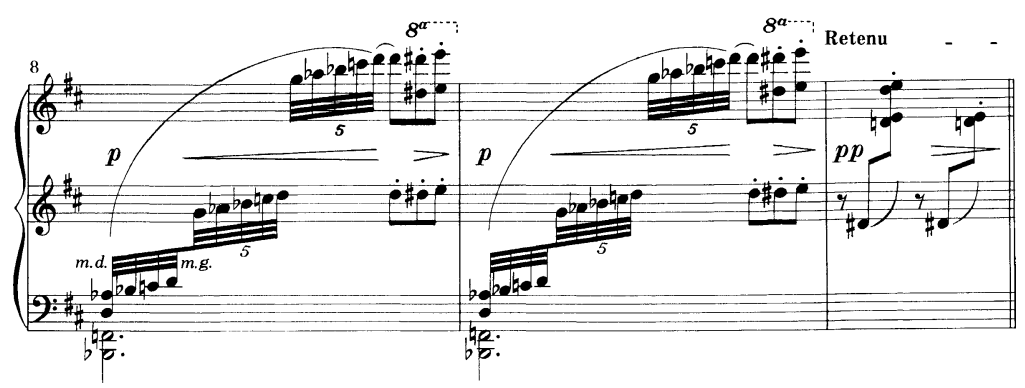

Beispiel 18: Claude Debussy, Préludes II, Nr. 8, T. 8-10

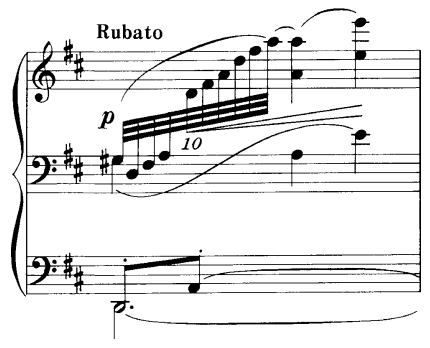

Beispiel 19: Claude Debussy, Préludes II, Nr. 8, T. 14

\subsection{Distanzielle Tonsysteme}

Tonsysteme, die die Oktave in gleiche oder periodisch alternierende Abstände aufteilen, können als distanziell bezeichnet werden. ${ }^{7}$ Unter den distanziellen Oktaveinteilungen spielen die Ganztonleiter und die alternierende Achtstufigkeit (Wechsel zwischen Halbton- und Ganztonschritt), die späteren Messiaen-Modi 1 und 2, im Impressionismus wohl die wichtigste Rolle. Einige ihrer Erscheinungsformen und Möglichkeiten zum hörenden Erschließen sollen im Folgenden beschrieben werden.

7 Vgl. Gárdonyi/Nordhoff 1990, 156. 


\subsubsection{Ganztonfelder}

Allgemeines

Die Ganztonleiter ist laut de la Motte die zweite Möglichkeit, das indonesische Tonsystem Slendro in der temperierten Zwölfstufigkeit darzustellen. ${ }^{8}$

Um mit Ganztonfeldern zu arbeiten, muss man sie zunächst einmal erkennen. Einen optischen Hinweis gibt dabei die relativ hohe Anzahl von Versetzungszeichen, die zur Darstellung ganztöniger Felder nötig sind: Unabhängig von der Generalvorzeichnung werden für mindestens zwei bzw. drei von sechs Tönen Versetzungszeichen benötigt. (Ein weiterer optischer Anhaltspunkt ist im Übrigen die oftmals >großterzige` oder 'großsekundige Auftragung wie in den Beispielen 20 (I, 2, T. 1-4) und 21 (II, 12, T. 53-54). Wegen der mitunter merkwürdigen Silben, die sich dadurch beim Solmisieren ergeben würden, und des oftmals nicht sehr deutlichen Grundtonbezugs erscheint die relative Solmisation hier weniger praktikabel.

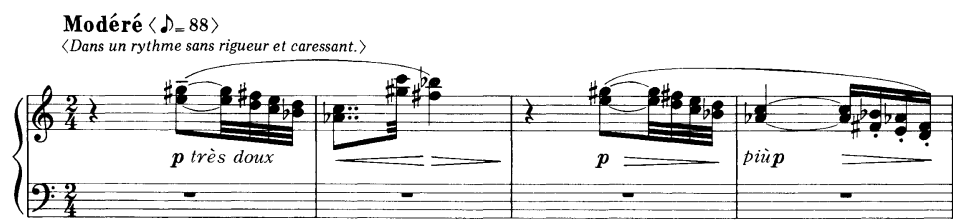

Beispiel 20: Claude Debussy, Préludes I, Nr. 2, T. 1-4

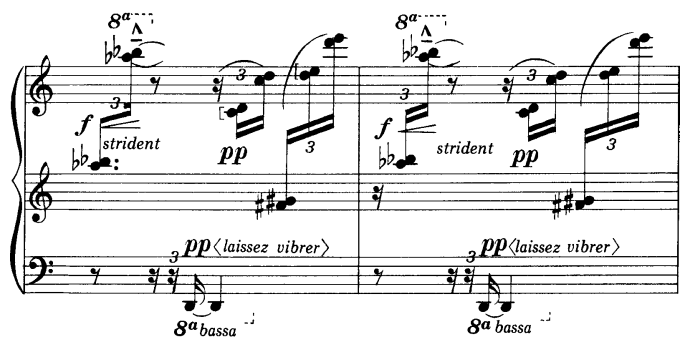

Beispiel 21:

Claude Debussy,

Préludes II,

Nr. 12 , T. $53-54$

Skalare Strukturen

Zum Einhören in weitere typisch ganztönige Fakturen eignet sich das Stück Voiles (Préludes I, Nr. 2), das gänzlich - mit Ausnahme von lediglich 6 Takten - auf Ganztonmaterial beruht. In den Terzmixturen der bereits zitierten Anfangstakte (Beispiel 20) kann bei langsamem Tempo abwechselnd die Ober- und Unterstimme auf Tonnamen gesungen werden, während die andere Stimme dazugespielt wird (wobei natürlich - der Klavierstimmung entsprechend - gleichstufig temperierte, nicht etwa reine, Großterzen entstehen sollen). Das Fortsetzen der realen Mixtur fördert dabei die Gewandtheit im Finden der Tonnamen und der zugehörigen Tasten.

8 La Motte 1976, 249f.; vgl. hierzu Anm. 2. 


\section{Nichtskalare Strukturen}

Beispiel 22 (I, 2, T. 58-61) zeigt in der linken Hand eine weitere typische Mehrklangsstruktur im ganztönigen Raum: Die Assoziation mit terzlosen halbverminderten Septakkorden liegt nahe. Hier liegen ebenfalls Mixturen vor, die jedoch nicht ausschließlich Versetzungsgrößen in Sekundschritten aufweisen. Auch sie können durch mehrstimmiges Üben (singen und dazuspielen) erarbeitet werden.

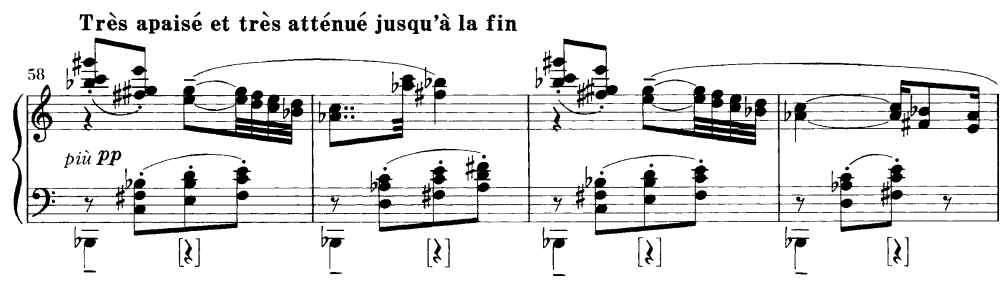

Beispiel 22: Claude Debussy, Préludes I, Nr. 2, T. 58-61

Anhand von Beispiel 23 (II, 11, T. 91-94) kann im Rahmen des Stimmumfangs Treffsicherheit im Bereich `Weitmelodikı geübt werden; singt man Ober- und Unterstimme und spielt dazu oktavreduziert die Zweitstimme, ergibt sich wiederum eine skalare Struktur.

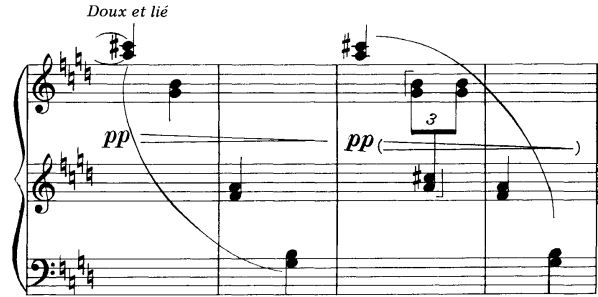

Beispiel 23: Claude Debussy, Préludes II, Nr. 11, T. 91-94

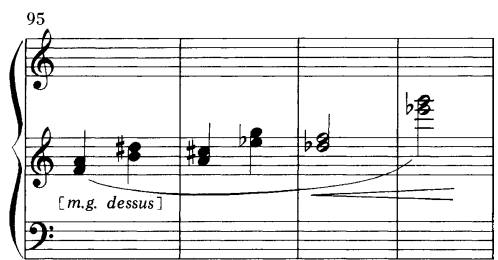

Beispiel 24: Claude Debussy, Préludes I, Nr. 11, T. 95-98

Beispiel 24 aus demselben Stück (T. 95-98) zeigt Intervallsprünge, die ersungen werden können. Auch bietet es sich bei den beiden zuletzt genannten Beispielen an, sie singend und spielend zu einer der oben gesehenen dreistimmigen Mixturen (übermäßiger Dreiklang oder shalbverminderter Klang) zu ergänzen.

Unterscheiden ganztöniger Felder von anderen Tonvorräten

Ganztönige Strukturen werden oft mit akustischen Feldern verwechselt, wahrscheinlich wegen der unteren ganztönigen Hälfte der akustischen Skala. Eine Übung zur Unterscheidung kann aus Beispiel 25 (I, 3, T. 15-16) erwachsen. In Takt 15 erklingt nur eine ganztönige Struktur (aus heses, ces, des und es). Der in Takt 16 hinzutretende Ton ges zeigt aber, dass es sich um einen Ausschnitt aus Heses- oder Ces-Akustisch handelt, nicht aus einem Ganztonfeld. Das Manipulieren des Zitats durch Singen und Spielen des Tones $g$ statt ges in der Unterstimme kann den klanglichen Unterschied verdeutlichen. 


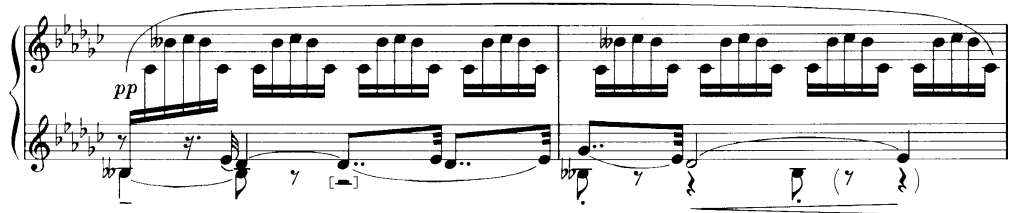

Beispiel 25: Claude Debussy, Préludes I, Nr. 3, T. 15-16

\subsubsection{Alternierende Achtstufigkeit}

Vorübungen

Die alternierende Achtstufigkeit oder Halbton-Ganztonskala ist nur begrenzt transponierbar. Beginnt man die Bildung der Skala vom Ton $c$ aus mit einem Halbtonschritt aufwärts, entsteht diejenige Struktur, die Messiaen als erste Transposition des 2. Modus bezeichnet hat. Von cis aus erhält man die zweite Transposition, von $d$ aus die dritte Transposition. Von dis aus konstruiert, entsteht derselbe Tonvorrat wie über $c{ }^{9}$

Für den im Umgang mit der alternierenden Achtstufigkeit noch Ungeübten bietet sich als Einstiegsübung an, die Skala in den drei möglichen Transpositionen zu singen. Diese Übung lässt sich dahingehend erweitern, dass in drei- bis vierstimmigen Mixturen (Beispiel 26) einzelne Stimmen gesungen und die anderen dazugespielt werden. Schließlich können die einzelnen Akkorde der Mixturen auch von unten nach oben durchgesungen werden. Dabei kann man einen Klang voraussingen und dann spielend nachkontrollieren. Indem der Übende überlegt, welcher Klang als nächstes folgt und wie er strukturiert sein muss, verschafft er sich Überblick über den Tonvorrat einer Transposition. Eine andere Übemöglichkeit besteht (gerade bei Vierklängen) darin, den Klang zuerst am Instrument anzuschlagen und dann die Töne herauszuhören und nachzusingen. So wird das Ohr im Erkennen von Klangtypen und Klangverbindungen geschult, die auch in Messiaens Kompositionen eine zentrale Rolle spielen. Ferner kann festgestellt werden, dass die Grundtöne der entstandenen Mixturklänge im Kleinterzabstand stehen.
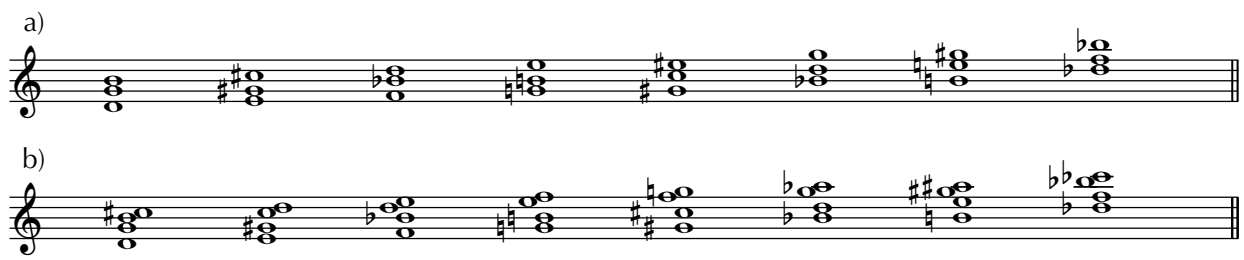

Beispiel 26: Mixturen in der alternierenden Achtstufigkeit

Mehrklangstrukturen

Beispiel 27 (II, 4, T. 60-64) lässt sich nutzen, um verschiedene Mehrklangstypen in ihrer Struktur zu erfassen und gleichzeitig der typisch kleinterzigen Abstände gewahr zu

9 Vgl. hierzu Messiaen 1966, 57. 
werden. Es kann - der Kompositionsweise entsprechend - in verschiedenen Schichten zur Übung herangezogen werden: Zunächst kann man die Klangtypen im mittleren und unteren System ermitteln, indem man die Akkorde von unten nach oben mit Tonnamen, auch mit `Funktionsnamen der einzelnen Akkordbestandteile (Beispiel des ersten Klangs in T. 62: 'Septime-Terz-Quinte-Grundton`), singt. Weiterhin kann der Lehrer mehrere Klänge unmittelbar nacheinander spielen, und die Studierenden hören die jeweiligen Grundtöne heraus oder singen sie hinein. Besondere Aufmerksamkeit gebührt der übermäßigen Undezime (T. 62 und T. 63, jeweils 2. Klang, Ton a) als typischer Hinzufügung.
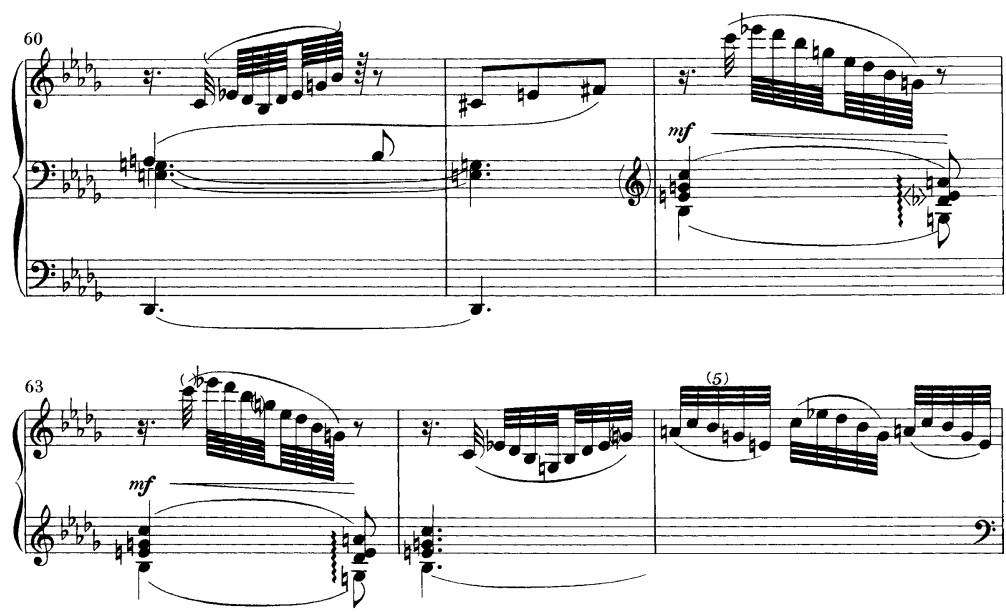

Beispiel 27: Claude Debussy, Préludes II, Nr. 4, T. 60-65

Die obere Schicht (System 1) kann erarbeitet werden, indem man die arpeggierten Klänge der Takte 60, 62 und 63 zuerst spielt und dann mit absoluten Tonnamen nachsingt. Danach kann man auch hier die Akkordtöne in ihrer Funktion singend benennen. Die Klänge der oberen Schicht lassen sich außerdem noch simultan, also nicht arpeggiert, anschlagen und singen.

Schließlich kann das ganze Beispiel gehört werden (eventuell ebenfalls mit simultan angeschlagenen Klängen der oberen Schicht). Dabei soll sich das Ohr (etwa wie das Auge beim Betrachten eines Vexierbilds) abwechselnd auf die untere und die obere Schicht konzentrieren und die Klänge identifizieren. Wie beim Einstudieren eines Klavierstücks, bei dem man an schwierigeren Stellen die 'Hände einzeln übt‘, kann man auch hierbei die schwer zu hörenden Momente herausgreifen und in der beschriebenen Art nochmals schichtweise erarbeiten.

Unterscheiden alternierend achtstufiger Felder von anderen Tonvorräten

An die vorangegangenen Übungsideen knüpft der Vorschlag an, den Klangeindruck der alternierenden Achtstufigkeit durch differenzierendes Hören von anderen Tonvorräten abzusichern. 
Nur zwei Töne unterscheiden die alternierende Achtstufigkeit von der akustischen Skala. Die folgende Übung kann die Fähigkeit trainieren, die beiden Tonvorräte voneinander abzugrenzen. Beispiel 28 (II, 3, T. 66-75) zeigt zunächst einen akustischen Undezimakkord (Dominantseptakkord mit übermäßiger Undezime), der auch in einem alternierend achtstufigen Tonvorrat aufgehen würde. Durch die Linie der Mittelstimme in Takt 68f. (namentlich den Ton c) werden kurzzeitig sowohl der akustische als auch der alternierend achtstufige Tonvorrat gesprengt. Die folgenden Takte können - die relativ große Präsenz der typisch akustischen Bestandteile legt dies nahe - abwechselnd im Original und in sakustischer Verfremdung ' gespielt werden, indem man die Töne e und $d$ durch diejenigen Töne ersetzt, die in Des-Akustisch enthalten sind.
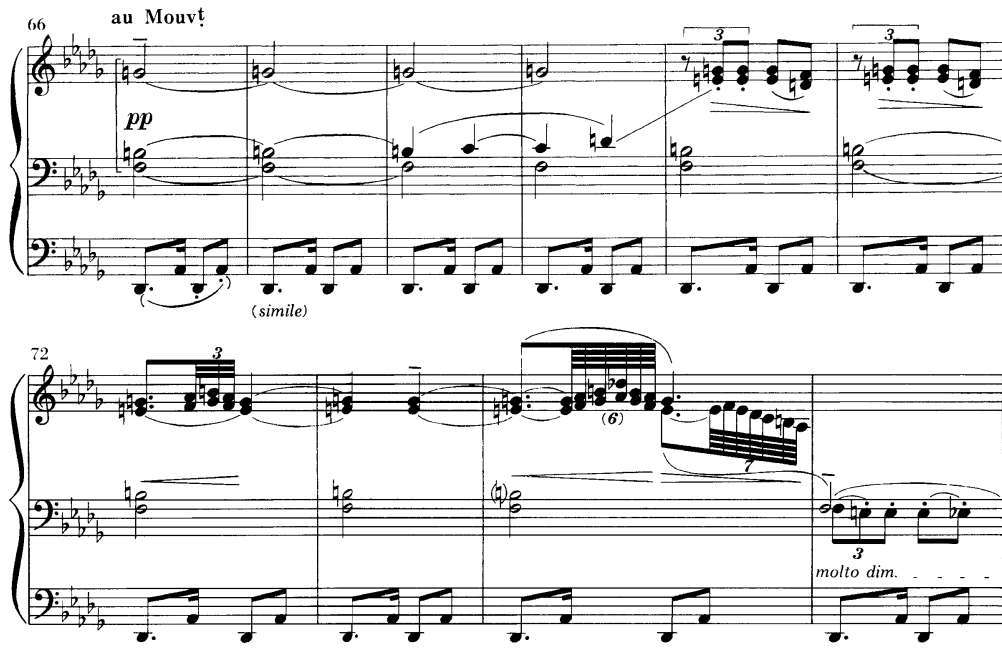

Beispiel 28: Claude Debussy, Préludes II, Nr. 3, T. 66-75

\section{MEHRKLANGSBILDUNGEN}

Als >Mehrklänge` werden im Folgenden diejenigen Klänge bezeichnet, die über den Septakkord hinausgehende Optionen enthalten, also ıMehr-als-Vierklänge`sind.

Dabei kann man zwischen den sehr häufigen akustischen Klangtypen, deren Töne skalar aufgetragen einige oder alle Töne einer akustischen Skala repräsentieren, und Klängen mit anderen Hinzufügungen unterscheiden. Akustische Mehrklänge können innerhalb eines längeren akustischen Feldes stehen, wie Beispiel 29 (I, 9, T. 113-116) zeigt. Hier entstehen über einem rhythmisch figurierten akustischen Nonakkord (Es 7/9) durch die Melodiestimme wechselnde Hinzufügungen der große Tredezime und der übermäßige Quarte. Somit sind alle Töne einer Es-akustischen Skala vertreten. Diese Faktur lädt zu der Übung ein, zunächst die Akkordschicht als Vierklang zu spielen, also unter Aussparung der None, und dann die Akkordanreicherungen hineinzusingen ${ }^{10}$ (quasi mit Fermate

10 Vgl. Stoiber 1995, 70. 
auf jedem Melodieton) und ihre unterschiedliche Schärfe wahrzunehmen. Durch das Transponieren dieser Übung wird die Flexibilität im Spielen und Singen geschult.

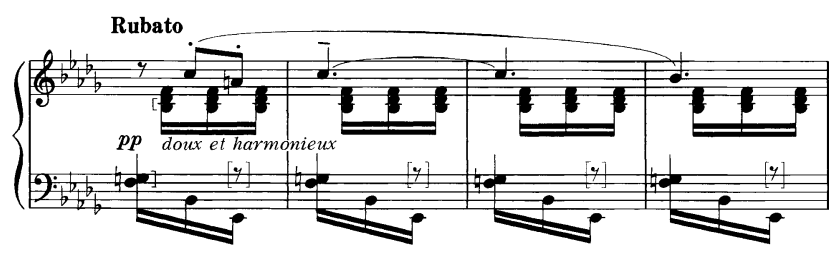

Beispiel 29: Claude Debussy, Préludes I, Nr. 9, T. 113-116

Beispiel 30 (II, 6, T. 39-42) bietet eine gute Übung für das Hören und Singen verschiedener Hinzufügungen. Zuerst kann die untere Schicht (notiert in den zwei unteren Systemen) durchgehört werden, in der bis auf die zweite Takthälfte von Takt 41 (akustischer Nonakkord) nur akustische Septakkorde vorkommen. In der bereits beschriebenen Weise soll der Studierende sie spielen und singen; dabei kann jede Struktur in ihrer Lage (Terz- oder Septlage) erfasst werden. Die punktierte Oberstimme erzeugt verschiedene Hinzufügungen. Sie sollen im langsamen Tempo in den liegenden Septakkord hineingesungen und auch hörend erfasst werden. Das Beispiel lässt sich dahingehend abwandeln, dass man jede der vorkommenden Hinzufügungen in jeden Klang hineinsingt, also etwa auch die übermäßige Quarte in den Es-Dur-Vierklang.

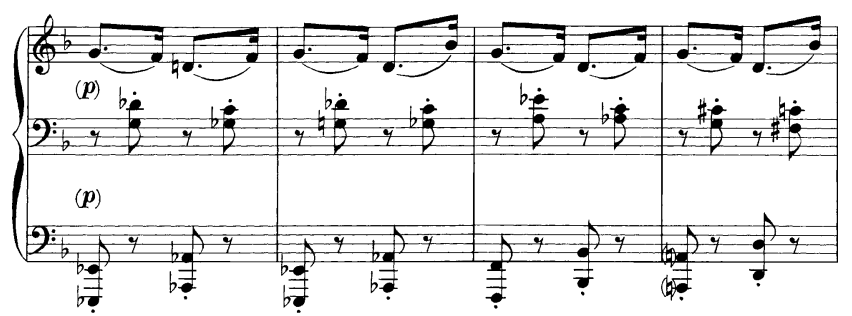

Beispiel 30: Claude Debussy, Préludes II, Nr. 6, T. 39-42

An Beispiel 31 (I, 6, T. 21-23) kann man sehr gut die besondere Klanglichkeit der >Doppelterzı, also der kleinen Terz zusätzlich zur großen Terz über dem Grundton (als übermäßige None oder kleine Dezime notiert) wahrnehmen. Hier soll ausschließlich das untere System betrachtet werden. Die Doppelterz (Ton e) tritt in der zweiten Takthälfte von Takt 21 zunächst nur figurativ auf und wird in die bereits im Klang vorhandene Terz $f$ weitergeführt. In der zweiten Hälfte von Takt 23 verwandelt sich der Ton e durch die veränderte Vierklangsbasis zur großen None, die in die Doppelterz $f$ weitergeführt wird. Diese bleibt bis zum Akkordwechsel bestehen. Auch diese Klänge können wiederum arpeggiert gesungen werden, ferner kann man über wechselnden Vierklangskernen die große None und die Doppelterz singend hinzufügen. Die Grundtonfolge Des- $D$ lädt weiterhin dazu ein, das Notenbeispiel improvisierend in Form einer realen Sequenz fortzusetzen, beispielsweise mit den Grundtönen $E s-E$ und den gleichen Hinzufügungen wie in Takt 23. 


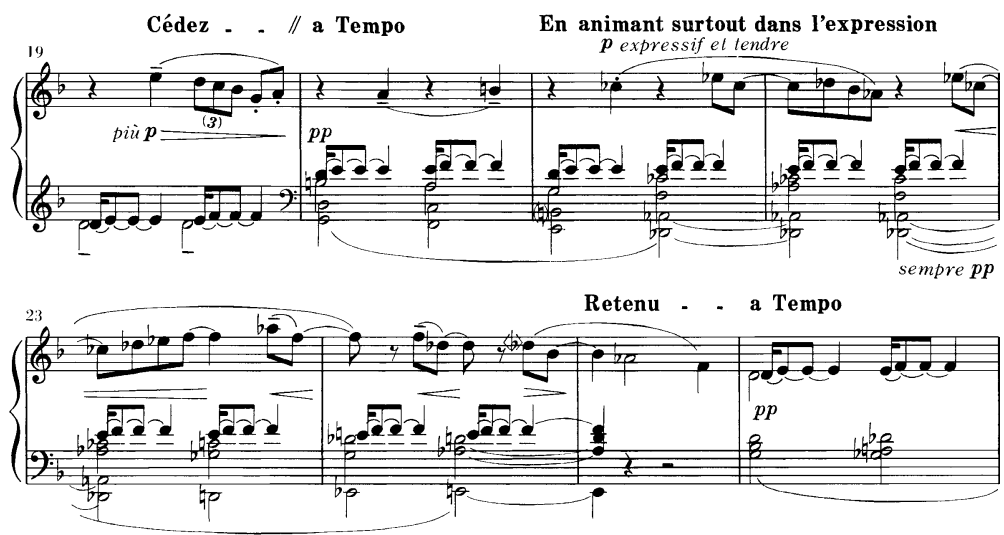

Beispiel 31: Claude Debussy, Préludes I, Nr. 6, T. 19-26

Auch an Beispiel 32 (II, 2, T. 15-16) lässt sich der besondere Charakter von Doppelterzklängen gut erfahren. Die fantasievolle Faktur regt hier ebenfalls dazu an, das Beispiel improvisierend weiterzuführen: Zuerst kann die reale Mixtur der Akkorde auf den TaktEinsen fortgesetzt werden, indem nach der Grundtonfolge cis-dis weitere ganztönige Schritte (oder andere Intervalle) gespielt und die Doppelterzklänge darüber arpeggiert gesungen werden; auch kann man die Klänge spielen und eine Oberstimme dazu singen, wie sie die Achtel des untersten Systems (höchste Stimme des Klaviersatzes) vorgeben: Hinzufügung von Septime und Doppelterz (T. 15) oder Quinte und Grundton (T. 16).

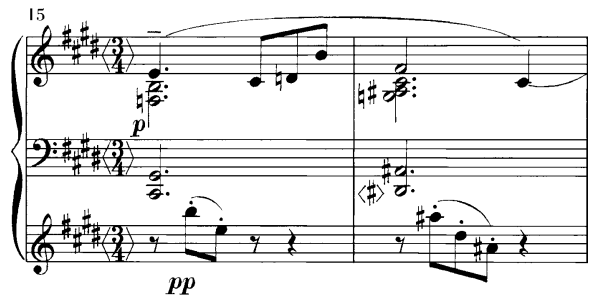

Beispiel 32: Claude Debussy, Préludes II, Nr. 2, T. 15-16

\section{MIXTUREN}

\subsection{Allgemeines}

Mixturen, also parallel geführte Mehrklänge ${ }^{11}$ kommen als farbliche Bereicherung von Melodielinien ('gesteigerte Einstimmigkeit`) oder als Begleitschichten vor. An ihnen lassen sich mehrere Dinge üben: Erstens können die Studierenden einzelne Klangtypen ermitteln und memorieren; zweitens können sie die Distanzen zwischen den Klängen bestimmen (keineswegs werden Mixturklänge immer nur schrittweise versetzt); drittens

11 Gárdonyi/Nordhoff 1990, 147. 
können sie die besondere Klanglichkeit verschiedener Mixturtypen wahrnehmen. Die folgenden Beispiele sind deshalb einerseits nach der Komplexität der Klangtypen, andererseits nach der Art der Mixtur (tonal, real) geordnet.

Hörübungen, die mit Hilfe eines jeden der Notenbeispiele durchführbar sind, bestehen darin, Beginn und Schluss des Mixtur-Abschnitts zu ermitteln und die Mixtur fortzusetzen, indem man die vorgegebene Struktur auf neue Tonhöhen versetzt weiterspielt und -singt.

\subsection{Tonale Mixturen}

Tonale Mixturen (Mixturen, die sich ausschließlich im Tonvorrat einer Tonart bewegen, wobei die Klangtypen sich den tonartlichen Gegebenheiten anpassen) sind häufig Dreiklangsmixturen. Sie stehen mitunter in einem archaisierenden programmatischen Zusammenhang oder haben zumindest eine entsprechende Klangwirkung.

Beispiel 33 (I, 10, T. 72-75) zeigt eine tonale Mixturmelodie aus grundstelligen Dreiklängen über einer Begleitschicht in Form eines figurierten Orgelpunktes. Zunächst können hier die Einzelklänge durchgehört und in Geschlecht und Lage identifiziert werden; dann kann man die Einzelstimmen solmisiert und absolut singen. Da es sich um eine tonale Mixtur handelt, lassen sich alle Töne im unalterierten Solmisationsbereich bzw. in der 0-Vorzeichen-Ebene ausdrücken.
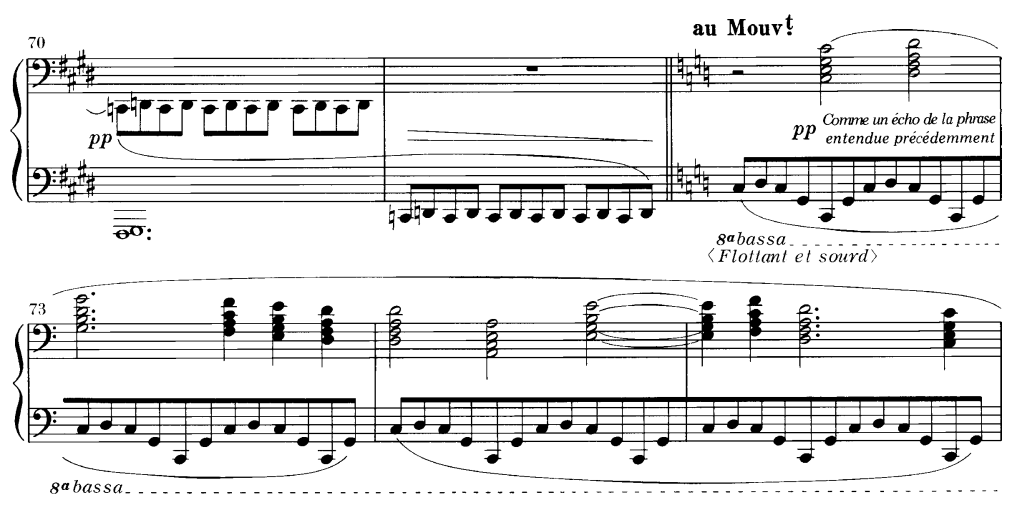

Beispiel 33: Claude Debussy, Préludes I, Nr. 10, T. 70-75

Beispiel 34 (I, 6, T. 29-31) zeigt eine Mixturschicht als Begleitstruktur zu einer Oberstimmenmelodie. Zunächst soll nur die Mixturschicht betrachtet werden. Nach der Identifikation der Einzelklänge als Sextakkorde soll der Studierende die tonale Ebene der Mixtur ermitteln und die Einzelklänge und Einzelstimmen solmisiert singen. Dann kann auch die Melodielinie des oberen Systems einbezogen werden, indem abwechselnd die eine Schicht gespielt und die andere dazu gesungen (die Mixturschicht arpeggiert) wird. Schließlich sollte man bei mehrmaligem Hören des Beispiels mit den umgebenden Takten den Beginn und vor allem den Schluss der Mixtur ermitteln können, der mit dem Wechsel von Sextakkorden zu Grundakkorden in Takt 31 zusammenfällt. 

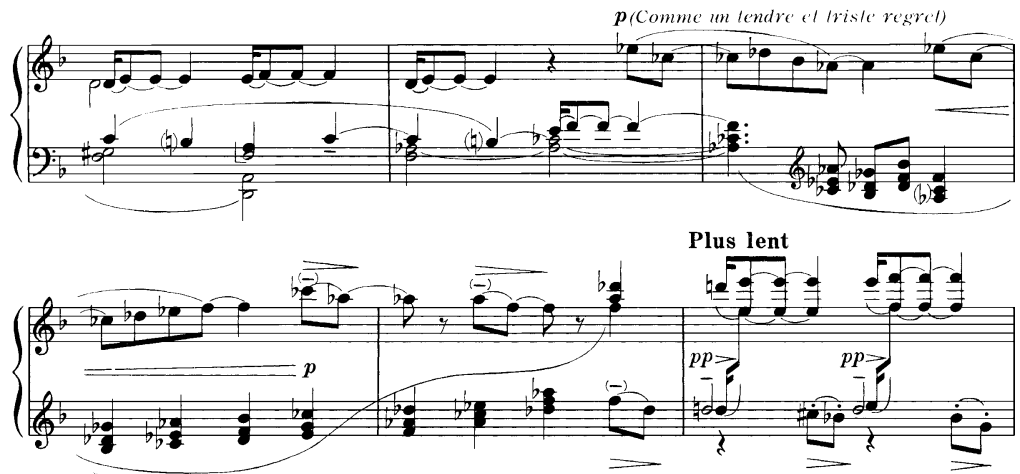

Beispiel 34: Claude Debussy, Préludes I, Nr. 6, T. 27-32

\subsection{Reale Mixturen}

\section{Vorbemerkung}

Bei realen Mixturen ist das Hören der Einzelklänge, sobald einmal der (gleichbleibende) Klangtyp ermittelt wurde, von minderer Bedeutung. Wichtiger ist hier das Hören der Entfernungen zwischen den einzelnen Klängen; da die Klänge von einer Tonart oder Tonalität losgelöst sind, sind auch die Entfernungen schwieriger zu erkennen als bei der tonalen Mixtur.

\section{Dreiklangsmixturen}

Insgesamt fällt auf, dass es sich bei nahezu allen Dreiklangsmixturen um Durdreiklangsmixturen handelt. Vergleichsweise leicht zu erfassen ist Beispiel 35 (I, 7, T. 15-16), erstens, weil sich mit Beginn der realen Mixtur auch die Faktur des Klaviersatzes ändert; zweitens, weil die Figurationen der Mixtur nur akkordeigene Töne beinhalten, so dass sich die Einzelklänge (Dur-Quartsextakkorde) leicht identifizieren lassen; drittens, weil innerhalb einer Phrase ausschließlich chromatische Fortschreitungen vorliegen. Hier kann der Studierende in die figurierten Klänge hinein die drei Einzelstimmen (Grundton, Terz und Quinte des Durdreiklangs) auf absolute Tonnamen und im Legato singen, so dass die chromatische Struktur erkannt wird; außerdem können die figurierten Klänge auch unfiguriert, also mit gleichzeitig angeschlagenen Tönen, gespielt werden, so dass ihre klangliche Wirkung memoriert wird.

In Beispiel 36 (I, 1, T. 21-24) sind verschiedene Terzgrößen neben anderen Intervallen vorhanden. An die Durdreiklangsmixturen schließen sich hier Mixturen aus übermäßigen Dreiklängen an, die einerseits durch das Hören und Singen des Arpeggios von der vorherigen Faktur unterschieden werden müssen; andererseits soll man hier die schrittweisen Distanzen (ganz- oder halbtönig) hören und unterscheiden. 


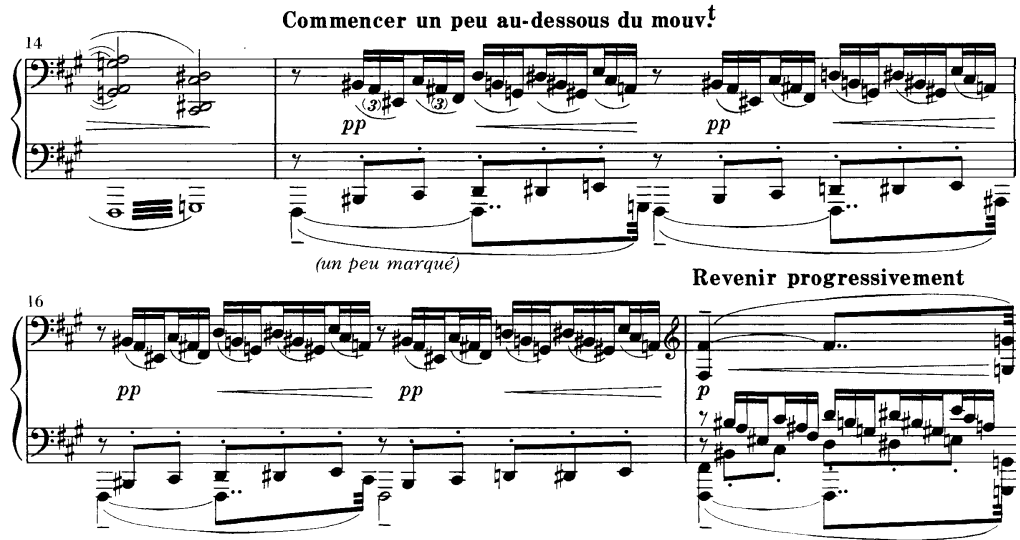

Beispiel 35: Claude Debussy, Préludes I, Nr. 7, T. 14-17
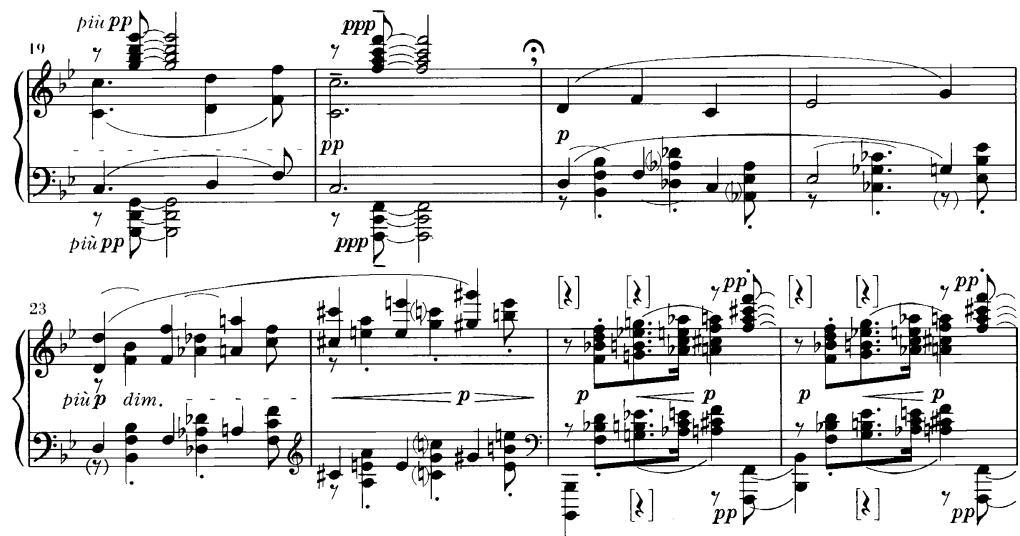

Beispiel 36: Claude Debussy, Préludes I, Nr. 1, T. 19-26

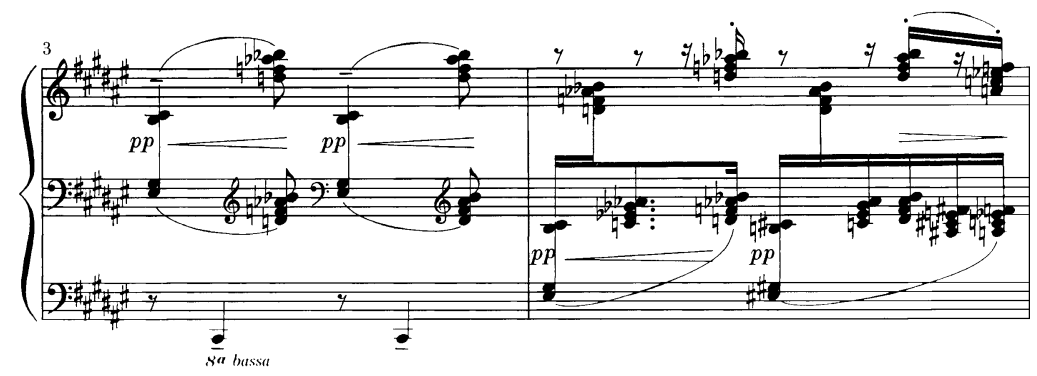

Beispiel 37: Claude Debussy, Préludes II, Nr. 7, T. 3-4 


\section{Vierklangsmixturen}

Bei den Mixturen aus Vierklängen finden sich besonders oft akustische Septakkorde und deren Umkehrungen. Beispiel 37 (II, 7, T. 3-4) zeigt eine reale Mixtur aus akustischen Quintsextakkorden. Nachdem durch Hören der Stelle im großformalen Zusammenhang ermittelt wurde, dass eine Mixturenfaktur vorliegt, kann man den parallel geführten Klangtyp durch arpeggiertes Singen herausfinden. Ob tatsächlich eine reale Mixtur vorliegt, kann überprüft werden, indem man jeden einzelnen Klang mit dem gefundenen Intervallmuster vergleicht, weiterhin indem man Einzelstimmen singt, dabei in jeden Klang den Grund-, Terzton etc. hineinsingt und darauf achtet, ob jede Stimme die gleiche melodische Linie ergibt. Auch die Distanzen zwischen den Einzelklängen (Terzen und Sekunden unterschiedlicher Qualität) lassen sich so ermitteln.

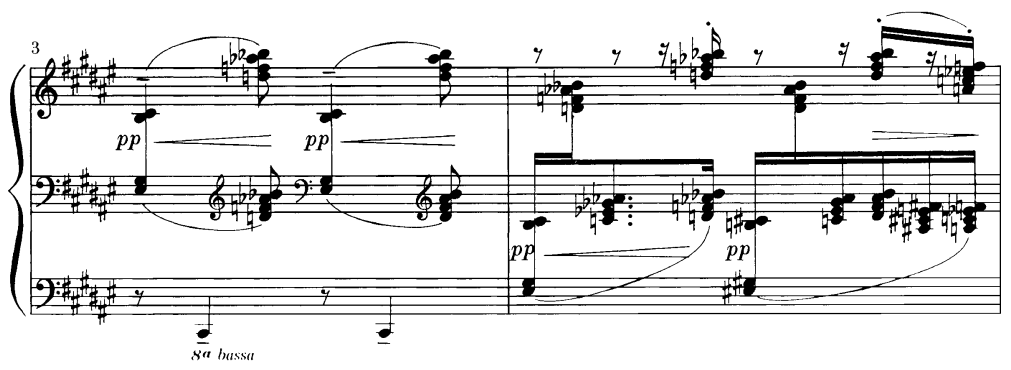

Beispiel 37: Claude Debussy, Préludes II, Nr. 7, T. 3-4

In gleicher Weise kann mit Beispiel 38 (II, 7, T. 31) gearbeitet werden, wo eine reale Mixtur aus akustischen Terzquartakkorden vorliegt. Hier wird man auf die distanzielle Anordnung der Klänge (Kleinterzkette der Grundtöne) hinweisen, ferner darauf, dass sich beim Singen der zweittiefsten und der darüberliegenden Stimme (je abwechselnd ein Ton) eine alternierend achtstufige Skala ergibt.

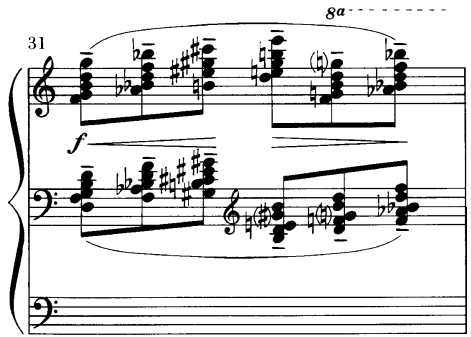

Beispiel 38: Claude Debussy, Préludes II, Nr. 7, T. 31

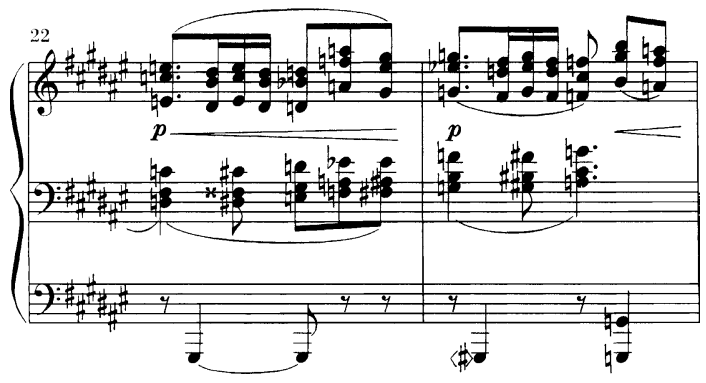

Beispiel 39: Claude Debussy, Préludes II, Nr. 7, T. 22-23

Beispiel 39 (II, 7, T. 22-23) zeigt im mittleren System eine Schicht aus einer realen Mixtur (akustische Septakkorde ohne Quinte), über der eine Schicht aus realen Mixturen in 
Großterzparallelen erklingt. Hier können zunächst die Einzelschichten in der beschriebenen Weise durchgehört werden. Auch kann man durch vertikales arpeggiertes Singen diejenigen Einzelklänge ermitteln, die sich durch das Zusammentreffen des akustischen Septakkords und der Großterzschicht als Hinzufügung ergeben (T. 22, 1. Achtel: akustischer Septakkord plus große None; 3. Achtel: akustischer Septakkord plus große Sexte plus kleine None etc.). Sobald der Studierende das Prinzip der Schichten aus realen Mixturen erkannt hat, entdeckt er auch leicht den Druckfehler, der sich in der verwendeten Ausgabe in Takt 22, 6. Achtel, befindet: Durch den Ton es anstelle von e würde die reale Mixtur kurzzeitig ohne erkennbaren (z. B. klanglichen) Grund unterbrochen.

\section{Mehrklangsmixturen}

Auch bei denjenigen Mixturen, deren Klänge neben der Septime weitere Hinzufügungen enthalten, ist wieder zweierlei interessant: der parallel geführte Klangtyp und die Schrittgröße der Parallelführung.

Die Einzelklänge in Beispiel 40 (II, 8, T. 4) kann man als unvollständige akustische Tredezimakkorde mit Septime im Bass verstehen. Dieses Beispiel ist wegen der figurierten Faktur gut hörend zu analysieren; ferner lässt es sich verfremden, indem man zu den einzelnen Klängen etwa noch den imaginären Grundton (z. B. T. 4, 4. Achtel: e) oder weitere Akkordtöne (z. B. None) hinzuspielt. Diese Klänge kann man auch unfiguriert vorspielen und singen lassen und sie mit den Klängen in Beispiel 41 (II, 4, T. 92-93) vergleichen. Schließlich können auch die dort vorliegenden Akkordtypen sowie deren Versetzungsgröße ermittelt werden.

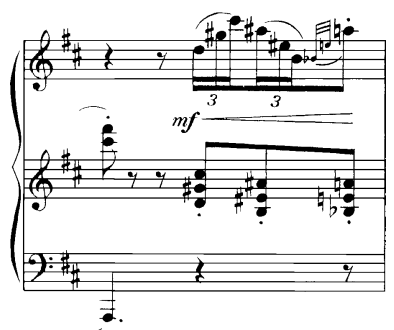

Beispiel 40: Claude Debussy, Préludes II, Nr. 8, T. 4

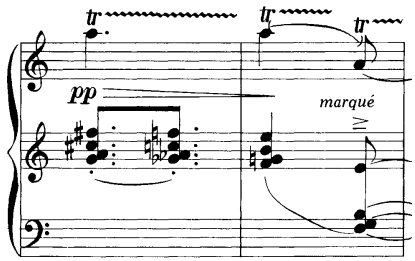

Beispiel 41: Claude Debussy, Préludes II, 4, T. 92-93

\section{SCHLUSSBEMERKUNG}

Der vorliegende Beitrag soll durch die Beschreibung möglicher Aufgabentypen dazu anregen, eigenständig neue Höraufgaben zu entwickeln und sich beim Hören kammermusikalischer wie orchestraler Fakturen immer wieder neu zu schulen. Die Beispiele lassen erahnen, dass die harmonischen Kompositionstechniken des Impressionismus für viele Studierende zu komplex sind, um ohne fundierte Tonsatzkenntnisse hörend erschlossen und begrifflich angemessen erfasst zu werden. Ideal wäre deshalb, wenn Tonsatz- und 
Gehörbildungsunterricht stilbezogen ineinandergriffen, so dass die analytisch gewonnenen Erkenntnisse hörend und spielend bzw. improvisierend nachvollzogen und die bei Hörübungen erhaltenen Klangeindrücke analytisch fundiert würden.

\section{Noten}

Debussy, Claude (1985), Préludes, Bde. I und II. Wiener Urtext Edition, Schott/Universal Edition, 2. Aufl., Wien.

\section{Literatur}

Bieri, Georg (1935): Die Lieder von Hugo Wolf, Phil. Dissertation Bern 1935, Leipzig: Typoskript.

La Motte, Diether de (1976), Harmonielehre, Kassel: Bärenreiter.

Hegyi, Erzsébet (1975), Solfège according to the Kodály-concept, Bd. I, Kecskemét: Zoltán Kodály Pedagogical Institute of Music.

Gárdonyi, Zsolt / Hubert Nordhoff (1990), Harmonik, Wolfenbüttel: Möseler.

Messiaen, Olivier (1966), Technik meiner musikalischen Sprache, Paris: Leduc 1966.

Schumacher, Rüdiger / Artur Simon / Margaret Karomi (1986), »Indonesien«, in: Musik in Geschichte und Gegenwart, 2. Aufl. hg. von Ludwig Finscher, Sachteil, Bd. 4, Kassel u. a: Bärenreiter, Sp. 766-851.

Stoiber, Franz Josef (1995), Gehörbildung, Tonsatz, Improvisation, Regensburg: ConBrio. 